\title{
Retroauricular Endoscope-Assisted Approach to the Neck: Early Experience in Latin America
}

\author{
Renan Bezerra Lira ${ }^{1}$ Thiago Celestino Chulam ${ }^{1}$ Yoon Woo Woo Koh ${ }^{2}$ Eun Chang Chang Choi ${ }^{2}$ \\ Luiz Paulo Kowalski ${ }^{1}$ \\ ${ }^{1}$ Department of Head and Neck Surgery and Otorhinolaryngology, \\ AC Camargo Cancer Center, São Paulo, SP, Brazil \\ 2 Department of Head and Neck Surgery and Otorhinolaryngology, \\ Younsei University College of Medicine, Seoul, Korea the Republic of \\ Address for correspondence Renan Bezerra Lira, MD, Department of \\ Head and Neck Surgery and Otorhinolaryngology, Rua Prof. Antonio \\ Prudente, 211, São Paulo 01509-900, SP, Brazil \\ (e-mail: renan.lira@accamargo.org.br).
}

Int Arch Otorhinolaryngol 2016;20:138-144.

\begin{abstract}
Keywords

- neck dissection

- thyroidectomy

- video-assisted surgery

- minimally invasive surgical procedures
\end{abstract}

Introduction There has been a significant increase in concern towards improving aesthetic and functional outcomes without compromising the oncologic effectiveness in head and neck surgery. In this subset, endoscope-assisted and robotic procedures allowed the development of new approaches to the neck, including the retroauricular access, which is now routinely used, especially in Korea.

Objectives This study aims to provide a descriptive analysis of our initial experience with retroauricular endoscope-assisted approach assessing feasibility, safety, and aesthetic results.

Methods Prospective analysis of the first 11 eligible patients submitted to retroauricular endoscope-assisted approach for neck procedures in the Head and Neck Surgery Department at AC Camargo Cancer Center.

Results A total of 18 patients were included in this study, comprising 7 supraomohyoid neck dissections, 8 submandibular gland excisions, 3 thyroid lobectomies, and one paraganglioma excision. There was no significant local complications, surgical accident, or need for conversion into conventional open procedure in this series.

Conclusion Our initial experience has shown us that this approach is feasible, safe, oncologically efficient, and applicable to selected cases, with a clear cosmetic benefit.

\section{Introduction}

In recent decades, head and neck oncologic surgery has been making remarkable progress with the development of various forms of minimally invasive surgical procedures and improvement in reconstruction techniques. These procedures have not only led to significant improvements in survival rates but also to increased satisfaction and quality of life of treated patients. ${ }^{1}$ However, a large number of patients are still submitted to extensive resections with or without neck dissection, and these can result in varying degrees of aesthetic and functional sequelae associated with numerous psychosocial repercussions. ${ }^{2}$

Head and neck surgery is characterized by the manipulation of delicate and important structures. Moreover, it has a very complex anatomy of multiple contiguous layers and cavities with difficult access because they are surrounded by bone and cartilage. Thus, except for nasosinusal and laryngeal lesions, minimally invasive head and neck approaches have been avoided until recent years because of concerns over exposure and adequate visualization of the operated sites. The purpose had been to avoid injury to received

July 15, 2015

accepted

December 6, 2015

published online

March 7, 2016
DOI http://dx.doi.org/

10.1055/s-0036-1578807. ISSN 1809-9777.
Copyright $\odot 2016$ by Thieme Publicações License terms

Ltda, Rio de Janeiro, Brazil

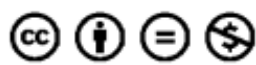


neurovascular structures since the instruments available that would facilitate these approaches were rudimentary and generally imprecise. ${ }^{3}$

On the other hand, there have been secular patient wishes and professional concerns in reducing functional and cosmetic morbidity without compromising oncological radicality. Several technological developments in endoscopy- and robotic-assisted procedures resulted in remarkable progress in minimally invasive procedures in all fields of surgical oncology, including head and neck surgery. ${ }^{4}$ Among these advances, endoscopic as well as DaVinci robotic system procedures have been incorporated by several surgical subspecialties. In selected cases, these surgical technologies eliminate the need for large visible neck incisions and provide superior functional and cosmetic results, having an acceptable cost and generating low complication rates that are equal or lower than those of classical procedures. However, some new complications of these minimally invasive techniques have been described. ${ }^{5-7}$

Although transoral robotic surgery (TORS) is now well established in head and neck oncology, it is not yet performed on a larger scale because of difficulties in the economic viability of these procedures in most centers around the world. Thus, the technology is currently available for a limited number of selected cases. Considering the economic reality of most countries, the alternative use of video-assisted endoscopic techniques seems to be a more feasible option for optimizing aesthetic and functional outcomes of selected patients with malignant and benign head and neck tumors. ${ }^{8}$

The search for treatment options that improve aesthetic and psychosocial outcomes has driven the development of different surgical approaches with better cosmetic results, including the transaxillary and retroauricular approaches for thyroidectomy, neck dissection, and resection of salivary gland tumors. ${ }^{9-18}$ In addition to the need for specific training, it is important to emphasize that some cases of brachial plexus injury were described among patients who underwent transaxillary thyroidectomy, and this discouraged surgeons from incorporating this approach into their practice. ${ }^{19}$ These limitations of transaxillary access led to the development of an alternative approach, the retroauricular access, which is now mainly used by Korean surgeons and a few Departments in North America. ${ }^{20}$ This access has the advantages of being located in an anatomical area that is familiar to head and neck surgeons, presenting similar results regarding cancer control (e.g., number of lymph nodes resected), reduced rates of post-operative complications, and better cosmetic results for patients. ${ }^{9,12,13,16,21}$ Furthermore, retroauricular approach has greater surgical safety than classical neck dissection incisions because, in the event of flap necrosis or dehiscence of the suture, large cervical vessels are not exposed, eliminating the consequent risk of catastrophic bleeding. ${ }^{13}$

The main object of this paper is to describe a pioneer experience in Latin America of a series of patients who underwent endoscope-assisted modified retroauricular approach.

\section{Methods}

Prospective analysis of the first 18 eligible patients that underwent surgery by the retroauricular endoscope-assisted approach for different head and neck tumors such as supraomohyoid neck dissections (SOHND), benign neck mass excision, submandibular gland excision, and thyroidectomy. All patients were treated at the Head and Neck Surgery and Otorhinolaryngology Department of our institution, from June 2014 to January 2015. This paper received approval from the Committee of Ethics in Research of our hospital. The inclusion criteria were as follows: patients with benign neck mass, submandibular gland tumors, unilateral thyroid suspicious or positive for malignancy nodules, or oral cavity proven squamous cell carcinoma without clinically metastatic lymph nodes ( $\mathrm{cNO}$ ) that were candidates for elective neck selective neck dissection of levels I-III. We excluded from this study patients with previous neck surgery or radiation and with metastatic disease (cN1-3).

Patient preparation for surgery was the same as that which is typically used for other neck surgeries performed under general anesthesia. The patient was positioned at the operating table with cervical extension and contralateral head rotation. The surgeon performs the retroauricular incision (-Fig. 1) and raises the subplatismal skin flap exposing the surgical field limited by neck midline, lower mandible border, omohyoid muscle, and esternocleidomastoid muscle, as described at Yonsei Medical Center Head and Neck Department in Seoul. ${ }^{15,16,22}$ During flap elevation, it is important to identify and preserve the great auricular nerve and external jugular vein. Then, a Thompson self-retaining retractor (Thompson Surgical Instruments, Traverse City, U.S.A.) was applied establishing the proper working space. Neck dissection (-Fig. 2), submandibular or neck mass excision was then performed with assistance of an ultrasonic scalpel (Ultracision ACE, Johnson \& Johnson, U.S.A.), vascular clips (Hem-olock, Teleflex, Morrisville, U.S.A.), a 10mm, 30-degree endoscope, and usual laparoscopic instruments (Grasper and Maryland forceps). The surgeon performed any dissections lateral to the carotid artery under direct vision using headlights, albeit without magnification tools, and regular surgical instruments, before introducing the endoscope. Routine dissection and preservation of facial marginal branch, vagus, hypoglossus, lingual, accessory, and phrenic nerves were

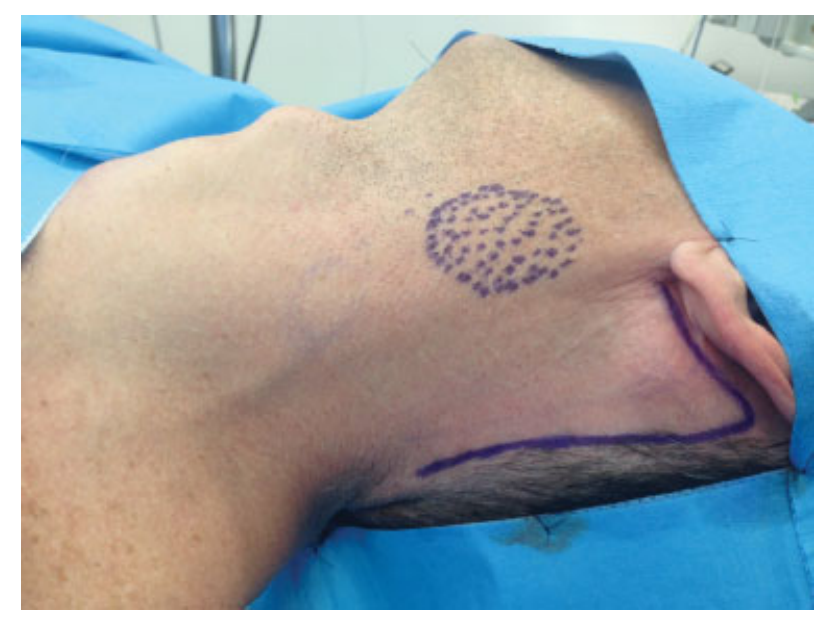

Fig. 1 Retroauricular incision planning. 


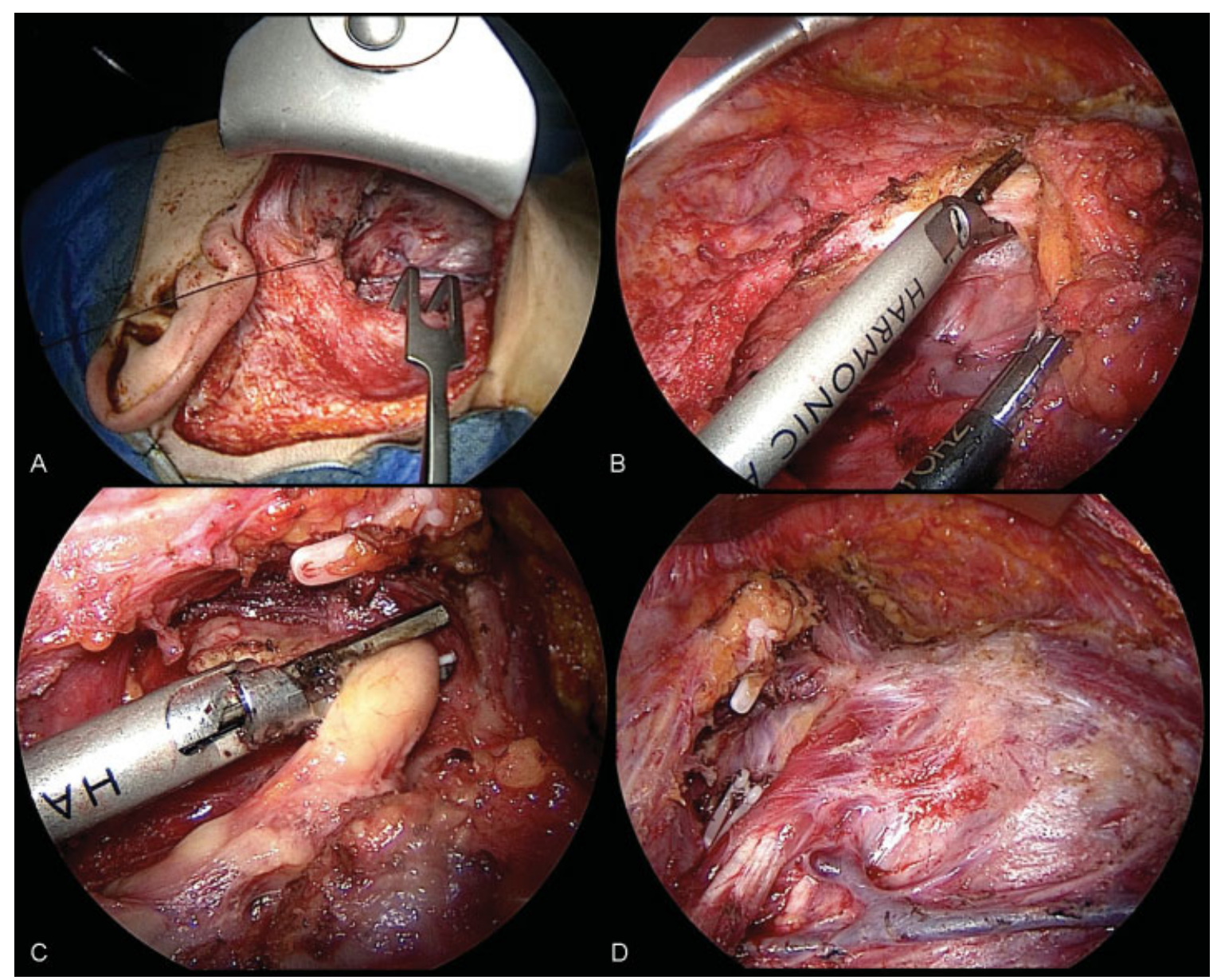

Fig. 2 Retroauricular endoscopy-assisted SOHND. (A) Overview of workspace; (B) Removal of levels II and III; (C) SMG duct being divided; (D) Final aspect of surgical field.

performed only for SOHND. Selective nerves were dissected and preserved for other the procedures, as needed. Closed aspirative drains (Blake, Ethicon Inc., Somerville, U.S.A.) were placed in all cases. The medical team discharged patients following the routine of the department for similar surgical procedures. All patients were followed at the Head and Neck Surgery outpatient clinic for a minimum of 30 days. At every post-operative visit, the surgeon evaluated the patients every post-operative visit for possible complications such as seroma, hematoma, surgical site infection, cranial nerve impairment, and skin flap dehiscence or necrosis. The frequency of these visits varied according to the surgical procedure performed and the patient's specific requirements. We also collected data regarding time to drain removal, which was performed when the drain output was less than $20 \mathrm{~mL} /$ day.

\section{Results}

Our study included a total of 18 patients, 6 women (33\%) and 12 men (67\%) with a mean age of 52.1 years (Range: $23-74$ years) (-Table 1). The surgical time was 30-75 minutes for flap raising, retractor placement and conventional dissections, and 30-105 minutes for endoscopy-assisted dissection and resection. Below, we describe this series of cases by type of surgical procedure performed.

\section{Vagal Paraganglioma Resection}

In our series, one case was of a vagal paraganglioma (pathological diagnosis) and the preoperative fine needle aspiration results were inconclusive; thus, we initiated the operation under the assumption that the neck mass was benign. This patient had a transient hypoglossal deficit that was highly manipulated due to the topographical characteristics of the lesion and vagus nerve paralysis with dysphonia and aspiration because the tumor was located at nerve and needed to be resected. There was no bleeding or infection. The duration of drainage was 6 days. The hospital stay was 3 days, as the patient required aspiration control with speech-swallowing therapy.

\section{Thyroid Lobectomy}

We performed retroauricular endoscopy-assisted hemithyroidectomy in three cases. The first one was a 66-year-old man, which was diagnosed with a $15 \mathrm{~mm}$ left parotid nodule, with fine needle aspiration biopsy (fNA) compatible with benign epithelial lesion. During pre-operative work-up, we diagnosed a left $12 \mathrm{~mm}$ thyroid nodule with a Bethesda class III cytopathology. He was then submitted to a left face-lift approach with subsequent partial parotidectomy with preservation of the facial nerve and its branches and left endoscopy-assisted thyroid lobectomy. The other two patients were one 69-year-old woman and one 52-year-old man, 
Retroauricular Endoscope-Assisted Approach Lira et al. 141

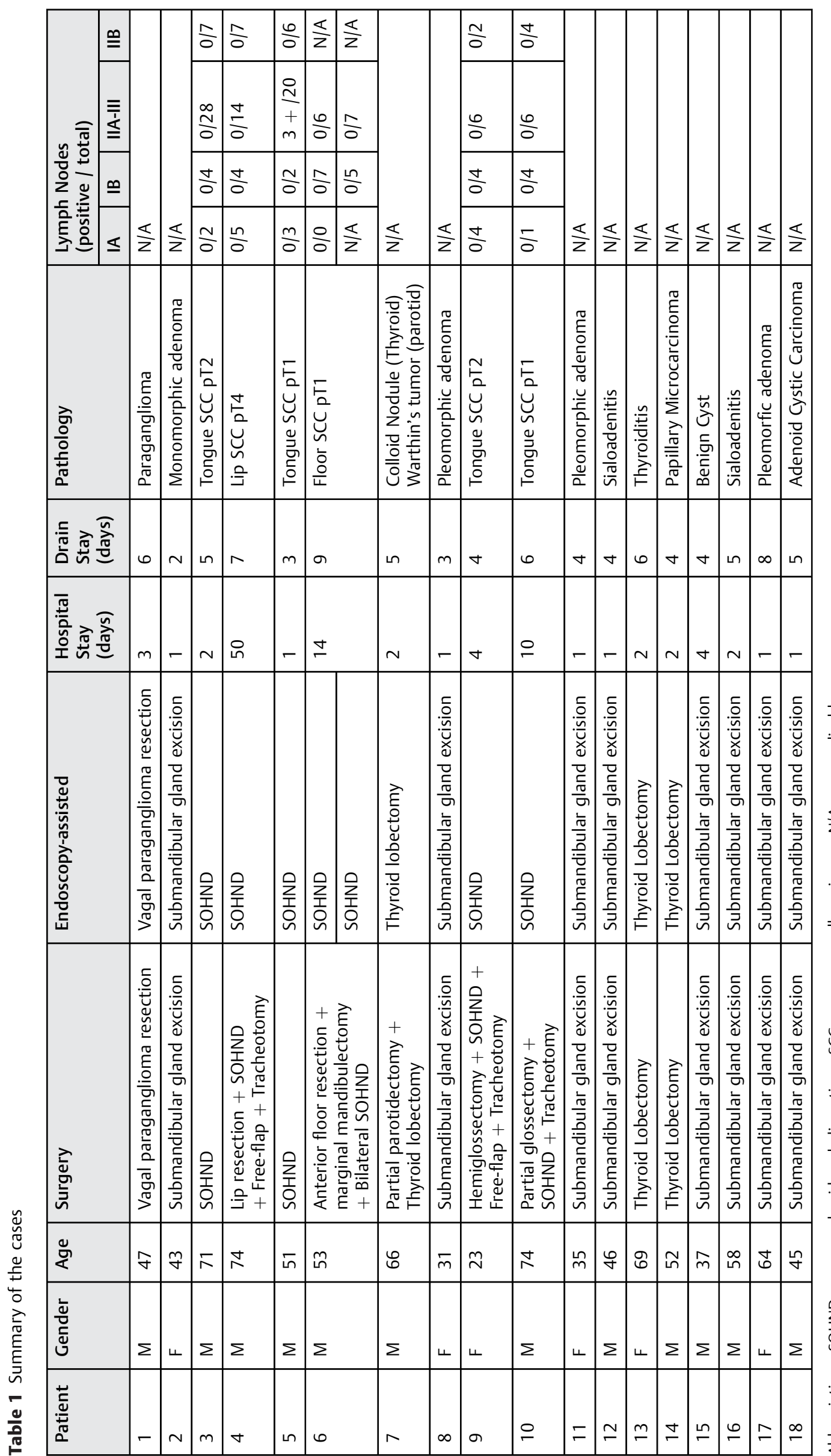


both with Bethesda class III unilateral thyroid nodule, which received retroauricular endoscopy-assisted hemithyroidectomy. We performed all three surgical procedures with no incidents, allowing good visualization and preservation of parathyroid glands and laryngeal nerves. There were no vocal cord palsy or hypocalcemia. The last patient presented with cervical hematoma immediately after extubation. We performed reoperation through the same incision, with endoscopy-assisted review and re-sealing of an anterior jugular vein. All three patients were discharged 48 hours after the surgical procedure. We removed the drains between 4 and 6 days after surgery (mean of 5 days). After pathological analysis, two patients were diagnosed with benign thyroid nodules and one patient with low risk papillary microcarcinoma.

\section{Supraomohyoid Neck Dissection}

We performed seven selective neck dissections I-III in 6 patients (one patient received bilateral dissection). All cases had oral cavity squamous cell carcinoma: one lip, one anterior floor, and four tongue lesions. Two of them underwent free flap reconstruction, using the same surgical incision. Three patients received temporary tracheotomy. There were no significant incidents or neck complications in these cases, aside from a small $(3 \times 8 \mathrm{~mm})$ skin flap necrosis. Two patients presented HB grade II marginal paresis. The number of resected lymph nodes ranged from 12 to 41 , with a mean of 22.5 node. Excluding the patient submitted to the bilateral $\mathrm{ND}$, where level IIb was not resected, the range was 15-41 and the mean 26.6 lymph nodes. The mean time of drain stay was 6.1 days (range 3-9 days). The patient submitted to lip resection and free-flap reconstruction had pulmonary sepsis with long hospital stay. The other patients had a mean hospital stay of 5.2 days.

\section{Submandibular Gland Excision}

There were eight cases of submandibular gland excision, of which four were women and four men (50\%). Seven patients had presumed benign diagnosis and one patient had a palpable $25 \mathrm{~mm}$ hard nodule with FNA showing undetermined cytology. All first seven cases confirmed benign pathology in the definitive pathology report (three pleomorphic adenomas, one monomorphic adenoma, one benign cyst, and two sialoadenitis) and the last one was diagnosed with cribiform adenoid cystic carcinoma with clear margins. We had no related flap necrosis, infection, or bleeding complications. Five out of eight cases (62.5\%) presented paresis HouseBrackmann Grade II of marginal mandibular branch with complete recovery within 30 days. There was no other type of neural deficit related to anatomical district (hypoglossal and lingual nerve). The mean duration of drainage was 4.3 days. Six patients were discharged in the first postoperative day, two in the second, and one patient stayed in the hospital for 4 days because of social issues (he was able to receive discharge one day after surgery).

Although our sample is heterogeneous, including patients from public and private health care systems, we were able to compare cost of submandibular gland excisions. For this, we reviewed hospital bills from three patients of our sample which were submitted to endoscopic SMG excision and from another three patients submitted to conventional SMG excision in the same period, all of them treated under private health insurance. The average costs were 4293 USD and 4153 USD, respectively.

\section{Discussion}

The advent of minimally invasive surgical techniques began in the 1980s, and these techniques have been employed in many surgical specialties, including pelvic, abdominal, thoracic, and, most recently, head and neck surgery ${ }^{21}$. In the field of head and neck surgery, the first endoscopically assisted operation was performed by Gagner in 1996. ${ }^{23,24}$

With the advent of endoscopic techniques and the subsequent advent of robotic procedures, the need for remote access to the neck through a transaxillary or retroauricular approach arose with the goal of improving the functional and aesthetic results of neck surgery without compromising the oncological results. The retroauricular approach for robotic and endoscopic surgery seems to provide a shorter and more direct route to the neck, requiring minimal dissection and providing an adequate workspace. Therefore, developing and refining the surgical techniques of endoscopic facelift thyroid and neck surgery is worthwhile.

Several limitations and concerns regarding this facelift approach for endoscope-assisted surgeries have prevented it from becoming commonly used worldwide. First of all, the endoscope and instruments used in this approach are controlled by two surgeons, and sometimes it is difficult to manipulate the instruments in a narrow space. ${ }^{5}$ Other issues with this technique are collisions between the operator and the assistant, making the operation somewhat uncomfortable, and limitations in the range of motion of the manually operated instruments. The rigid, straight nature of the endoscope and the other instruments further contributes to the limited ability to manipulate them. The lack of a third arm, as is used in robotic techniques, can sometimes render the operation difficult and time-consuming. More limited vision through the 2-dimensional image is expected from the endoscopic surgery when compared with the 3-dimensional image provided by the robotic surgery imaging systems. Another important disadvantage of endoscopic neck dissection is need for the operator to have sufficient skill in handling the endoscopic. Surgeons must be accustomed to the endoscopic surgical view and the handling of the instruments, such as the endoscope, endoscopic dissector, and harmonic scalpel. ${ }^{17}$ Nonetheless, we witnessed clear improvements in surgical time and resourcefulness from the first procedure to the last ones during the learning curve within this process.

In this study, we report the first cases of endoscopyassisted neck surgery using retroauricular or facelift approach in Latin America. Most of the published data regarding these techniques have come from Asia, with smaller contributions from Europe ${ }^{25}$ and the USA. ${ }^{26,27}$ In these centers, retroauricular and facelift approaches have been more commonly used with DaVinci system for robotic neck 


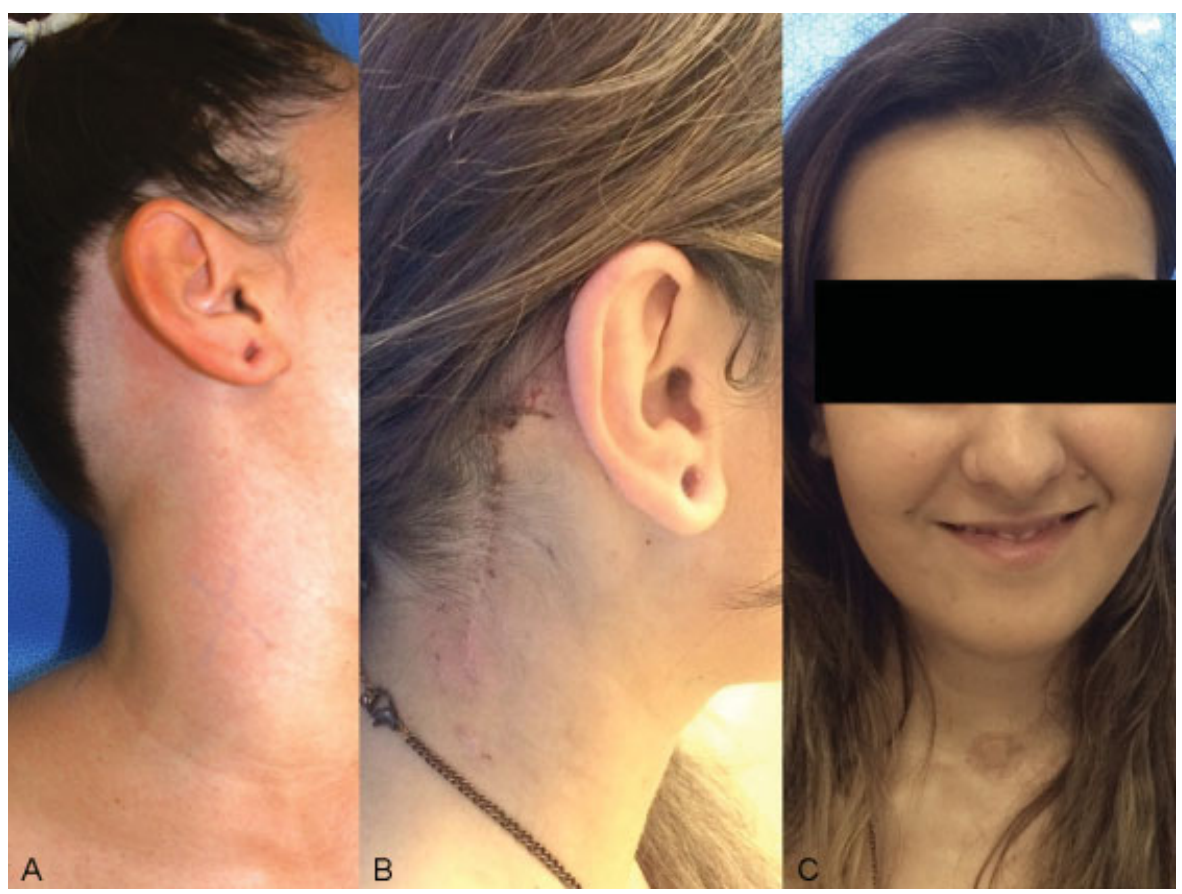

Fig. 3 Retroauricular endoscopy-assisted SOHND combined with hemi-glossectomy and free-flap reconstruction. (A) Preparation; (B,C) Retroauricular scar and cosmetic result in 14th post-operative day.

dissections, thyroid surgery and a wide range of other neck procedures.

In our sample, we observed no significant complications related to the approach or the surgical technique. No procedures were converted to open surgery and the significant neurovascular structures were preserved in all cases. There was no report of seroma or surgical site infection, but one patient had a hematoma following extubation. The incidence of low grade marginal nerve paresis was considered acceptable when compared with that of conventional procedures and when compared with those of previous published studies. ${ }^{16,17,28}$ This confirms the safety of this approach with or without use of robotic system, as we observed no increase in the incidence of complications when compared to the classical approach.

Although our small sample size and short follow-up period did not allow us to confirm the oncological effectiveness of this method, the number of lymph nodes retrieved in our sample was similar to those published for previous studies of classic or retroauricular SOHND. ${ }^{16,17,29,30}$ Another indirect indicator of the oncological effectiveness of this method is the final aspect of the surgical field after neck dissection, which is essentially the same for endoscopy-assisted neck surgery as for conventional SOHND. At an appropriate time, we will publish the oncological results of our experience with retroauricular approach, comparing with conventional surgery.

Regarding cosmetic results, there is a clear benefit of the retroauricular approach to neck surgery, especially as compared with conventional neck dissection and SMG excision, because the scar becomes hidden in the hair line with the retroauricular approach (-Fig. 3). A patient satisfaction score analysis performed in a prospective study by Lee et $\mathrm{al}^{16}$ showed a significant benefit of the retroauricular approach when compared with conventional neck dissection.

In regards to costs, Yoo et al reported that the mean cost of endoscopic thyroidectomy was $\$ 829$, or 8 times less expensive than robotic thyroidectomy. Furthermore, not all institutions have DaVinci systems, and most hospitals, particularly in developing countries, cannot afford to purchase such an expensive device. ${ }^{31}$ In our study, although cost comparison is not one of objectives, no important increase could be observed when compared costs of our conventional and endoscopic surgeries. Therefore, the endoscopic approach should not be considered out of date and is worth developing.

\section{Conclusion}

Our initial experience with an endoscopic approach to neck surgery has shown that this approach is feasible, safe, oncologically effective, and can be used in selected cases with a clear cosmetic benefit, even without the use of robotic DaVinci system. Thus, this is an option for hospitals that do not yet have access to the robotic system. Further analysis of a larger study sample is necessary to confirm the advantages of this endoscopic approach to neck surgery. In particular, a study with a more specific selection of cases and more thorough evaluations of costs, patient satisfaction, and learning curve for performing surgery by this approach should be performed.

\section{References}

1 Dziegielewski PT, Teknos TN, Durmus K, et al. Transoral robotic surgery for oropharyngeal cancer: long-term quality of life and 
functional outcomes. JAMA Otolaryngol Head Neck Surg 2013; 139(11):1099-1108

2 Weymuller EA, Yueh B, Deleyiannis FW, Kuntz AL, Alsarraf R, Coltrera MD. Quality of life in patients with head and neck cancer: lessons learned from 549 prospectively evaluated patients. Arch Otolaryngol Head Neck Surg 2000;126(3):329-335, discussion 335-336

3 Brunaud L, Germain A, Zarnegar R, Klein M, Ayav A, Bresler L. Robotic thyroid surgery using a gasless transaxillary approach: cosmetic improvement or improved quality of surgical dissection? J Vis Surg 2010;147(6):e399-e402

4 Goh HKC, Ng YH, Teo DTW. Minimally invasive surgery for head and neck cancer. Lancet Oncol 2010;11(3):281-286

5 van Loon JWL, Smeele LE, Hilgers FJM, van den Brekel MWM. Outcome of transoral robotic surgery for stage I-II oropharyngeal cancer. Eur Arch Otorhinolaryngol 2015;272(1):175-183

6 Ozer E, Alvarez B, Kakarala K, Durmus K, Teknos TN, Carrau RL. Clinical outcomes of transoral robotic supraglottic laryngectomy. Head Neck 2013;35(8):1158-1161

7 Chen MM, Roman SA, Kraus DH, Sosa JA, Judson BL. Transoral Robotic Surgery: A Population-Level Analysis. Otolaryngol Head Neck Surg 2014;150(6):968-975

8 Fan S, Liang FY, Chen WL, et al. Minimally invasive selective neck dissection: a prospective study of endoscopically assisted dissection via a small submandibular approach in $\mathrm{CT}\left(1-2 \_\mathrm{N}(0)\right.$ oral squamous cell carcinoma. Ann Surg Oncol 2014;21(12):3876-3881

9 Tae K, Ji YB, Song CM, Jeong JH, Cho SH, Lee SH. Robotic selective neck dissection by a postauricular facelift approach: comparison with conventional neck dissection. Otolaryngol Head Neck Surg 2014;150(3):394-400

10 Shin YS, Choi EC, Kim C-H, Koh YW. Robot-assisted selective neck dissection combined with facelift parotidectomy in parotid cancer. Head Neck 2014;36(4):592-595

11 Sun GH, Peress L, Pynnonen MA. Systematic review and meta-analysis of robotic vs conventional thyroidectomy approaches for thyroid disease. Otolaryngol Head Neck Surg 2014;150(4):520-532

12 Seup Kim B, Kang KH, Park SJ. Robotic modified radical neck dissection by bilateral axillary breast approach for papillary thyroid carcinoma with lateral neck metastasis. Head Neck 2015;37(1):37-45

13 Park YM, Holsinger FC, Kim WS, et al. Robot-assisted selective neck dissection of levels II to $\mathrm{V}$ via a modified facelift or retroauricular approach. Otolaryngol Head Neck Surg 2013;148(5):778-785

14 Lee J, Chung WY. Robotic thyroidectomy and neck dissection: past, present, and future. Cancer J 2013;19(2):151-161

15 Lee HS, Lee D, Koo YC, Shin HA, Koh YW, Choi EC. Endoscopic resection of upper neck masses via retroauricular approach is feasible with excellent cosmetic outcomes. J Oral Maxillofac Surg 2013;71(3):520-527

16 Lee HS, Kim WS, Hong HJ, et al. Robot-assisted Supraomohyoid neck dissection via a modified face-lift or retroauricular approach in early-stage cNO squamous cell carcinoma of the oral cavity: a comparative study with conventional technique. Ann Surg Oncol 2012;19(12):3871-3878
17 Byeon HK, Holsinger FC, Koh YW, et al. Endoscopic supraomohyoid neck dissection via a retroauricular or modified facelift approach: preliminary results. Head Neck 2014;36(3):425-430

18 Blanco RGF, Boahene K. Robotic-assisted skull base surgery: preclinical study. J Laparoendosc Adv Surg Tech A 2013;23(9): 776-782

19 Davis SF, Abdel Khalek M, Giles J, Fox C, Lirette L, Kandil E. Detection and prevention of impending brachial plexus injury secondary to arm positioning using ulnar nerve somatosensory evoked potentials during transaxillary approach for thyroid lobectomy. Am J Electroneurodiagn Technol 2011;51(4):274-279

20 Choi EC, Koh YW. Endoscopic and Robotic Neck Surgery. Seoul: Joo Sub Song; 2013

21 Lee J, Kwon IS, Bae EH, Chung WY. Comparative analysis of oncological outcomes and quality of life after robotic versus conventional open thyroidectomy with modified radical neck dissection in patients with papillary thyroid carcinoma and lateral neck node metastases. J Clin Endocrinol Metab 2013;98(7): 2701-2708

22 Koh YW, Chung WY, Hong HJ, et al. Robot-assisted selective neck dissection via modified face-lift approach for early oral tongue cancer: a video demonstration. Ann Surg Oncol 2012;19(4): 1334-1335

23 Dhiman SV, Inabnet WB. Minimally invasive surgery for thyroid diseases and thyroid cancer. J Surg Oncol 2008;97(8): 665-668

24 Gagner M. Endoscopic subtotal parathyroidectomy in patients with primary hyperparathyroidism. Br J Surg 1996;83(6):875

25 Arribas-Garcia I, Alcalá-Galiano A. Facelift approach for neck dissection in squamous cell carcinoma of the lateral border of the tongue: application of this novel aesthetic technique in head and neck oncologic surgery. J Craniofac Surg 2014;25(3): 1019-1020

26 Terris DJ, Singer MC. Robotic facelift thyroidectomy: Facilitating remote access surgery. Head Neck 2012;34(5):746-747

27 Greer Albergotti W, Kenneth Byrd J, De Almeida JR, Kim S, Duvvuri U. Robot-assisted level II-IV neck dissection through a modified facelift incision: initial North American experience. Int J Med Robot 2014;10(4):391-396

28 Lee HS, Kim D, Lee SY, et al. Robot-assisted versus endoscopic submandibular gland resection via retroauricular approach: a prospective nonrandomized study. Br J Oral Maxillofac Surg 2014;52(2):179-184

29 Patel SG, Amit M, Yen TC, et al; International Consortium for Outcome Research (ICOR) in Head and Neck Cancer. Lymph node density in oral cavity cancer: results of the International Consortium for Outcomes Research. Br J Cancer 2013;109(8):2087-2095

30 Friedman M, Lim JW, Dickey W, et al. Quantification of lymph nodes in selective neck dissection. Laryngoscope 1999;109(3): 368-370

31 Yoo H, Chae BJ, Park HS, et al. Comparison of surgical outcomes between endoscopic and robotic thyroidectomy. J Surg Oncol 2012;105(7):705-708 